\title{
Vehicle Handling Evaluation Models Using Artificial Neural Networks
}

\author{
Rui $\mathrm{Yu}^{1}$ and Xiaohui $\mathrm{Xia}^{2}$ \\ ${ }^{1 .}$ Changzhou Institute of Engineering Technology, Changzhou, Jiangsu 213164, \\ China; \\ 2. Changzhou College of Information Technology, Changzhou, Jiangsu 213164, \\ China \\ 13951206589@163.com
}

\begin{abstract}
Stability of vehicle handling is of great importance for measuring the safety of vehicles. According to the current evaluation method, this crucial property is usually determined by technicians using existing impact factors and the evaluation is always generated by subjective judgments because there are no united criterion that can simplify the evaluation. However, this evaluation process is very hard to achieve because of the large scale of independent variables. Here, we aim at presenting a novel method based on artificial neural networks (ANNs) to aid the evaluation process for the tests of stability of vehicle handling. We set different impact factors of the tests as the independent variables, while the scores of the tests were set as the dependent variables. Using the existing data, we trained it using linear predictor, general regression neural network (GRNN) and multi-layer feedforward network (MLFN) during the machine learning process. Results show that ANN models can be used for aiding the subjective evaluation of stability in vehicle engineering. Our research can offer a novel insight for the vehicle evaluation in future studies.
\end{abstract}

Keywords: Stability; Vehicle Handling; artificial neural network; subjective evaluation models

\section{Introduction}

Vehicle handling and stability are important factors affecting vehicle active safety [15]. To relevant workers, how to assess the stability of vehicle handling is a crucial work for ensuring the security. However, because the stability can be easily effected by various factors, we still do not have a united and accepted evaluation system. Therefore, we aim at finding out a effective and convenient model or system for stability evaluation in order to work out a subjective assessment system based on machine learning approaches [6-9]. Our study can effectively address the difficulties in relevant evaluations.

To simulate the subjective evaluation results of technicians, we decided to use artificial neural networks (ANNs) to mimic the non-linear relationship in people's mind. A series of models for snake-like test, input test of steering wheel angle step, input test of steering wheel angle pulse, test of steering agility and test of stable circle were developed to evaluate the scores of the stabilities of vehicles. We used the Results show that ANN models can effectively and robustly give the correct evaluation results after the machine learning process of ANN models based on the existing data and subjective evaluation results provided by technicians. 


\section{Artificial Neural Network}

Artificial neural network (ANN) is a machine learning system based on non-linear fitting with [10-13]. An ANN model consists of various nodes (which are what we regard as neuron). In the field of biology, there are cyanpses that on the edge of every neuron. The strength a cyanpse is what we call the weight. The powerful function of ANN models should not be seen as the function of each neuron, but should be seen as the whole network. Only by using the whole network made up of certain of neurons, can ANN models give good performances and results after the machine learning process set from its algorithms.

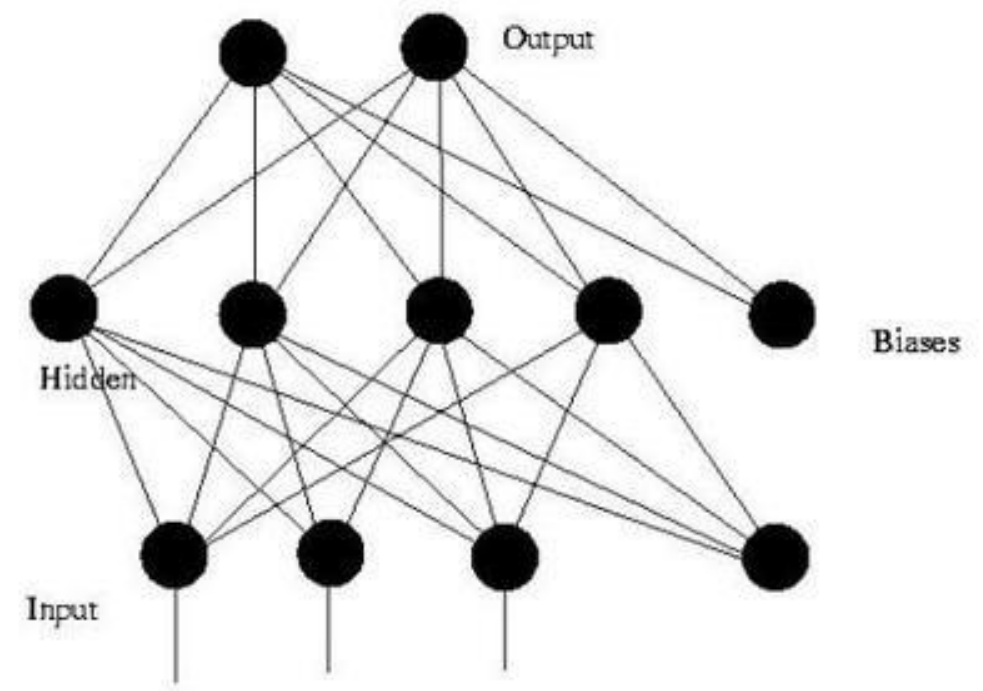

Figure 1. General Structure of an Artificial Neural Network

Figure 1 is a schematic structure of an ANN model, where the biases is used for extent the dimension of an ANN model for the sake of ensuring the normal running of the network. Under the most of the circumstances, a general ANN model consists of the input layer, the output layer and the hidden layer. Input layer is made up of independent variables. The number of nodes (or neurons) is decided by the number of input independent variables. Hidden layer is a layer for calculating the weights from the input layer. Users usually can choose the number of nodes (or neurons) in order to obtain the best component of an ANN model. Similar to the input layer, the number of nodes (or neurons) in the output layer is decided by the number of output dependent variables in practical applications.

\section{Models Establishment}

Our target of the research was to establish different models in different aspects for the evaluation of stability of vehicle handling respectively. Therefore, we separated the groups of the study objects into five aspects: snake-like test, input test of steering wheel angle pulse, test of stable circle, and the overall evaluation. In each aspect, we used general regression neural network (GRNN) $[14,15]$ and multi-layer feedforward neural network (MLFN) $[16,17]$ to establish the target models. In four different aspects, we used different independent variables for the sake of being close to reality. Here, we respectively present the modeling and calculating results of four aspects by using the data provided by Y. Bai, from Jilin University [18]. 


\subsection{Models for Snake-Like Test}

In the model development for the evaluation of snake-like test.We used the angle of steering wheel, average yaw rate, average angle of the vehicle body and average lateral acceleration as the independent variables in training process, meanwhile we set the subjective score as the dependent variable. In practical applications, this subjective scores are determined by technicians according to the sub-scores of the average yaw rate and the average steering angular velocity. In these models, we aim at using ANN models to obtain the exact scores directly. Table 1 shows the results of the ANN model for the evaluation of snake-like test.

Table 1. Results of Models for Snake-like Test

\begin{tabular}{cccc}
\hline ANN Model & $\begin{array}{c}\text { Trained } \\
\text { Samples }\end{array}$ & $\begin{array}{c}\text { Tested } \\
\text { Samples }\end{array}$ & $\begin{array}{c}\text { Average } \\
\text { RMS Error }\end{array}$ \\
\hline Linear Regression & 7 & 5 & 1.39 \\
GRNN & 7 & 5 & 0.96 \\
MLFN with 2 Nodes & 7 & 5 & 2.52 \\
MLFN with 3 Nodes & 7 & 5 & 175.68 \\
MLFN with 4 Nodes & 7 & 5 & 1.80 \\
MLFN with 5 Nodes & 7 & 5 & 172.82 \\
\hline
\end{tabular}

Table 1 shows that the GRNN model could generate the best responses, with a lowest RMS error (0.96). Results presented by Table 1 show that the GRNN model is the best model during our computational experiments.

\subsection{Models for the Input Test of Steering Wheel Angle Pulse}

In the model development for the input test of steering wheel angle pulse, we used the resonant frequency, resonant peak level and the phase lag as the independent variables in training process, meanwhile we set the subjective score as the dependent variable. In practical applications, this subjective scores are determined by technicians according to the sub-scores of the resonant frequency, resonant peak level and the phase lag. In these models, we aim at using ANN models to obtain the exact scores directly. Table 2 shows the results of the ANN model for the evaluation of the input test of steering wheel angle pulse.

Table 2. Results of Models for the Input Test of Steering Wheel Angle Pulse

\begin{tabular}{cccc}
\hline ANN Model & $\begin{array}{c}\text { Trained } \\
\text { Samples }\end{array}$ & $\begin{array}{c}\text { Tested } \\
\text { Samples }\end{array}$ & $\begin{array}{c}\text { Average } \\
\text { RMS Error }\end{array}$ \\
\hline Linear Regression & 7 & 5 & 0.84 \\
GRNN & 7 & 5 & 0.30 \\
MLFN with 2 Nodes & 7 & 5 & 8.48 \\
MLFN with 3 Nodes & 7 & 5 & 0.29 \\
MLFN with 4 Nodes & 7 & 5 & 2.77 \\
MLFN with 5 Nodes & 7 & 5 & 3.60 \\
\hline
\end{tabular}

Table 2 shows that the GRNN model and MLFN model with three nodes could generate the best responses, with lowest RMS errors ( 0.30 and 0.29 respectively). Results presented by Table 2 show that the GRNN model is the best model during our computational experiments. 


\subsection{Models for the Test of Stable Circle}

In the model development for the test of stable circle, we used the acceleration of neutral steering point, understeer degree and the degree of body roll as the independent variables in training process, meanwhile we set the subjective score as the dependent variable. In practical applications, this subjective scores are determined by technicians according to the sub-scores of the neutral steering point, acceleration of neutral steering point and the understeer degree. In these models, we aim at using ANN models to obtain the exact scores directly. Table 3 shows the results of the ANN model for the evaluation of the test of stable circle.

\section{Table 3. Results of Models for the Evaluation of the Test of Stable Circle}

\begin{tabular}{cccc}
\hline ANN Model & $\begin{array}{c}\text { Trained } \\
\text { Samples }\end{array}$ & $\begin{array}{c}\text { Tested } \\
\text { Samples }\end{array}$ & $\begin{array}{c}\text { Average } \\
\text { RMS Error }\end{array}$ \\
\hline Linear Regression & 7 & 5 & 2.14 \\
GRNN & 7 & 5 & 0.45 \\
MLFN with 2 Nodes & 7 & 5 & 0.48 \\
MLFN with 3 Nodes & 7 & 5 & 1.31 \\
MLFN with 4 Nodes & 7 & 5 & 1.98 \\
MLFN with 5 Nodes & 7 & 5 & 2.65 \\
\hline
\end{tabular}

Table 3 shows that the GRNN model could generate the best responses, with a lowest RMS error (0.45). Results presented by Table 3 show that the GRNN model is the best model during our computational experiments.

\subsection{Models for the Overall Evolution}

To sum up all the impact factors of the stability of vehicle handling, we also used an overall model for the integrated evaluation. We used the response time of yaw angle, resonant frequency, resonant peak level, phase lag, turning lateral acceleration of neutral point, understeer degree, degree of body roll, average turn angle, average yaw angle, average friction of steering wheel and maximum effect of the steering wheel as the independent variables, where as the overall evaluation score was set as the dependent variable. Results are shown in Table 4.

Table 4. Results of Models for the Overall Evolution

\begin{tabular}{cccc}
\hline ANN Model & $\begin{array}{c}\text { Trained } \\
\text { Samples }\end{array}$ & $\begin{array}{c}\text { Tested } \\
\text { Samples }\end{array}$ & $\begin{array}{c}\text { Average } \\
\text { RMS Error }\end{array}$ \\
\hline Linear Regression & 12 & 5 & 5.63 \\
GRNN & 12 & 5 & 4.11 \\
MLFN with 2 Nodes & 12 & 5 & 4.39 \\
MLFN with 3 Nodes & 12 & 5 & 2.23 \\
MLFN with 4 Nodes & 12 & 5 & 2.45 \\
MLFN with 5 Nodes & 12 & 5 & 4.78 \\
\hline
\end{tabular}

Table 4 shows that the MLFN model with 3 nodes could generate the best responses, with a lowest RMS error (2.23). Results presented by Table 4 show that the MLFN model under the condition of three nodes in the hidden layer is the best model during our computational experiments. 


\section{Results and Discussion}

Here, we use the classical method created by reference [19] to present the results of the best ANN models of the research presented in Section 3. For making comparison, we plotted the regression line in each figure.

\subsection{Models for Snake-Like Test}
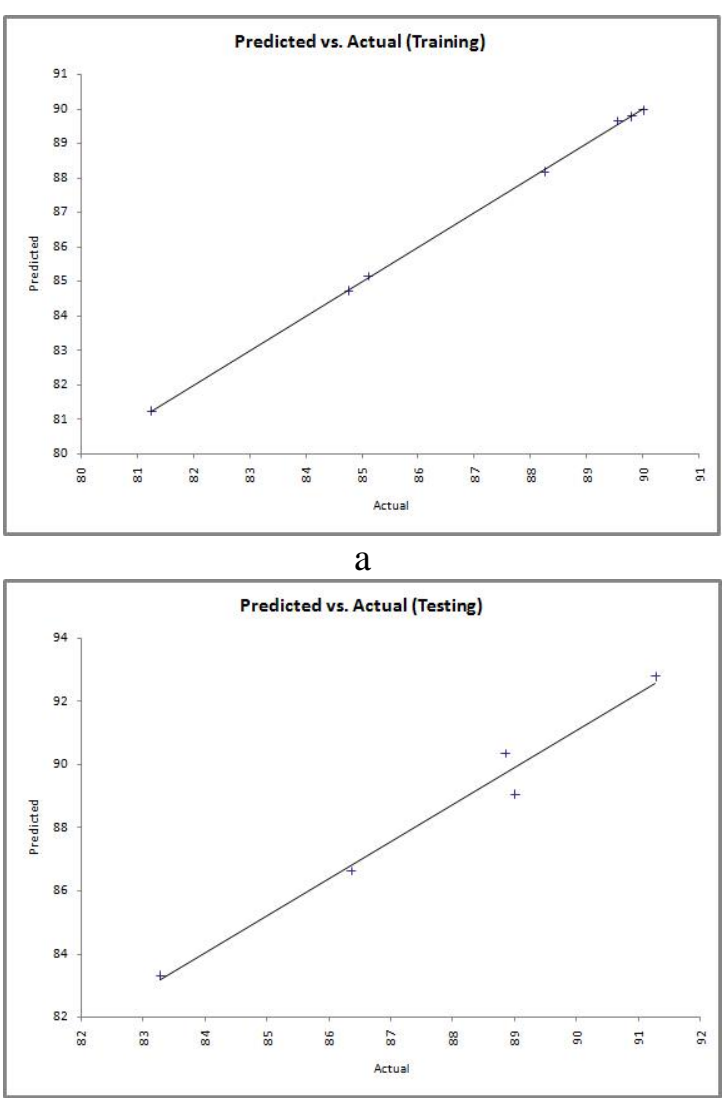

$\mathrm{b}$

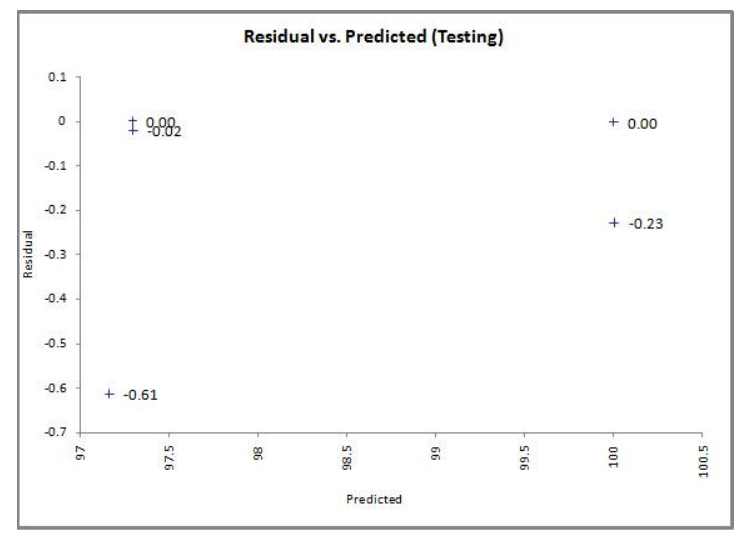

c

Figure 2. Training and Testing Results of GRNN Model (a) Predicted Values Versus Actual Values after the Training Process; (b) Predicted Values Versus Actual Values after the Testing Process; (c) Residual Values Versus Predicted Values in Testing Process 
Figure 2 shows shat the training and testing results are robust, indicating that the GRNN model is very effective for the evaluation in snake-like test for vehicles.

\subsection{Models for the Input Test of Steering Wheel Angle Pulse}

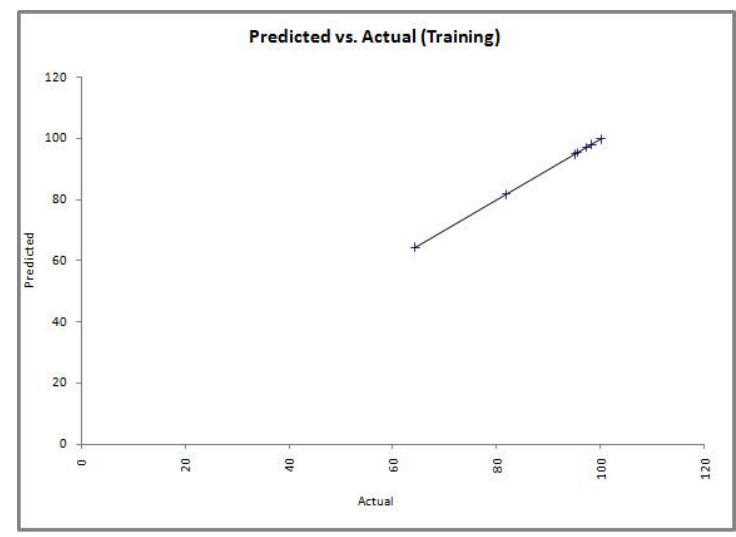

a

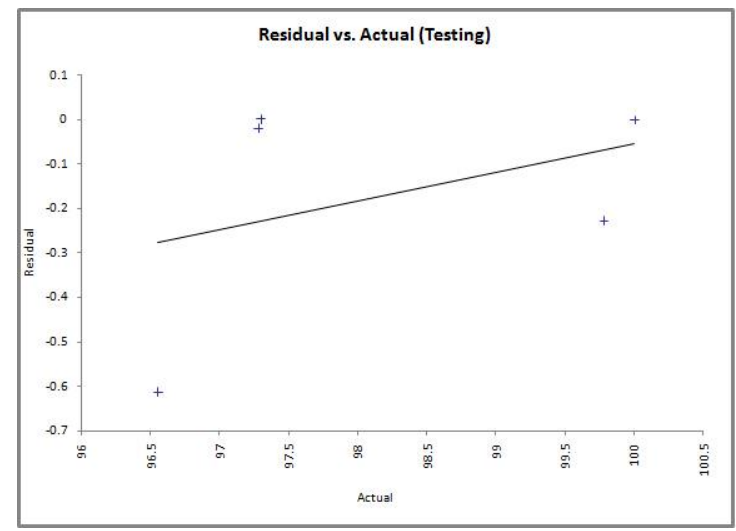

b

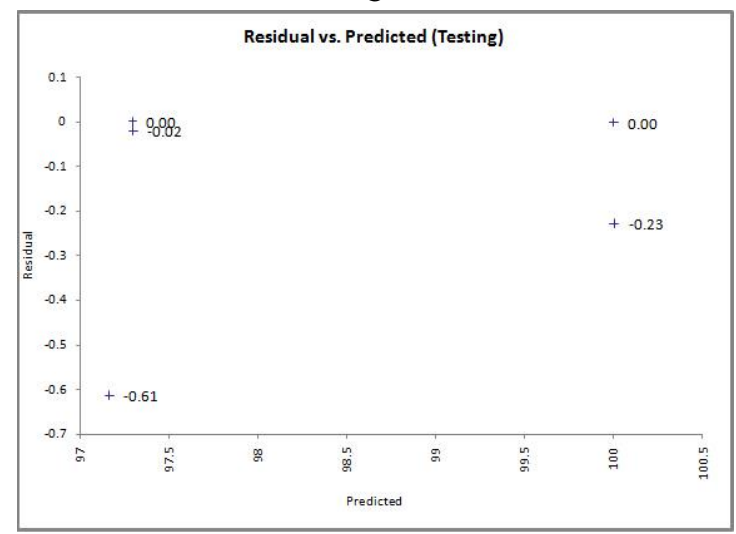

$\mathrm{c}$

Figure 3. Training and Testing Results of GRNN Model; (a) Predicted Values Versus Actual Values after the Training Process; (b) Predicted Values Versus Actual Values after the Testing Process; (c) Residual Values Versus Predicted Values in Testing Process

Figure 3 shows shat the training and testing results are robust, indicating that the GRNN model is very effective for the evaluation in the input test of steering wheel angle pulse for vehicles. 
4.3 Models for the Test of Stable Circle

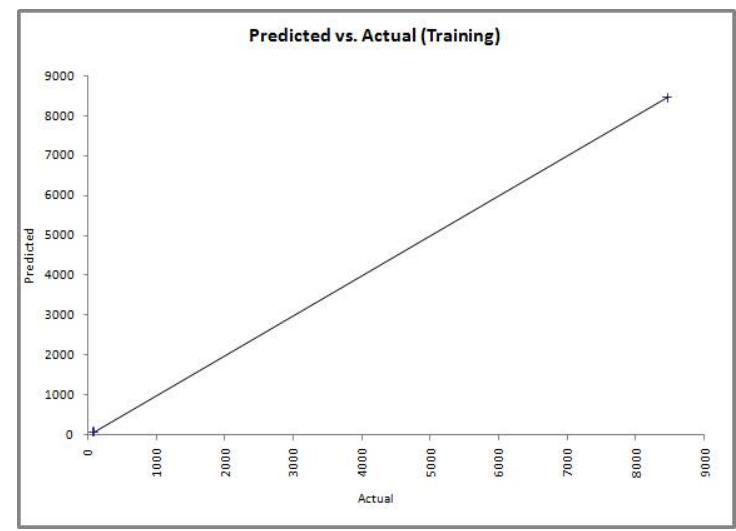

a

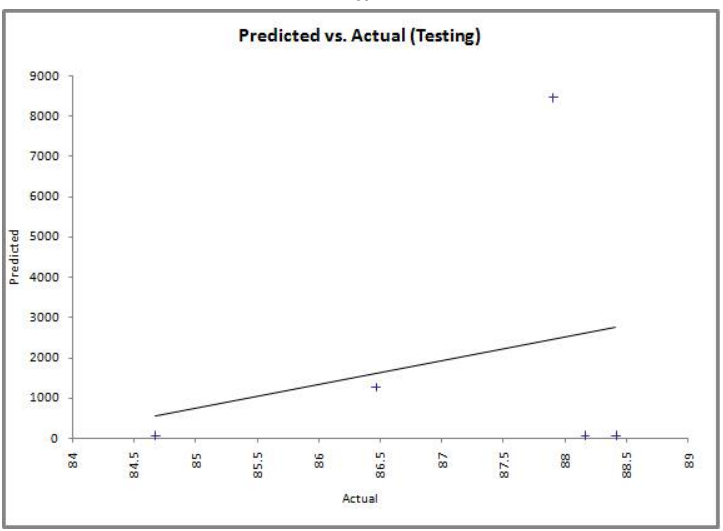

b

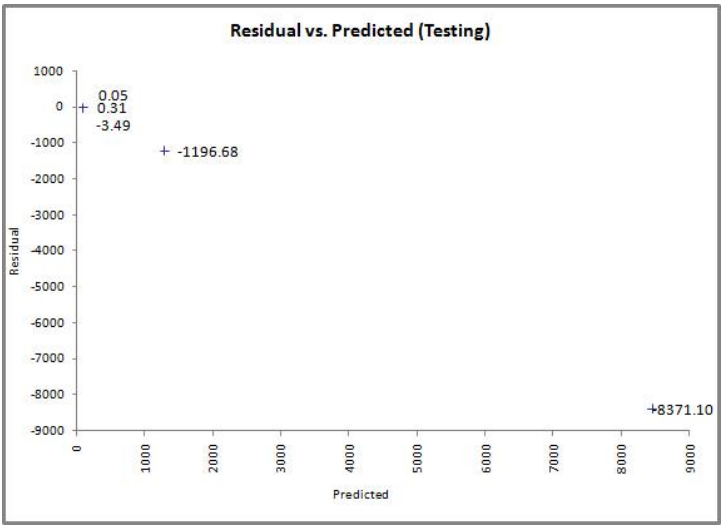

$\mathrm{c}$

Figure 4. Training and Testing Results of GRNN Model; (a) Predicted Values Versus Actual Values after the Training Process; (b) Predicted Values Versus Actual Values after the Testing Process; (c) Residual Values Versus Predicted Values in Testing process

Figure 4 shows shat the training and testing results are robust, indicating that the GRNN model is very effective for the evaluation in the test of stable circle for vehicles. Nevertheless, there are still some of the testing results that not quite suit for the actual values. We consider that this deviation is generated by the fluctuation of ANN models. For making further discussions, we will try more models that can perfectly suit for the actual results of the evaluation for the test of stable circle. 


\subsection{Models for the Overall Evolution}

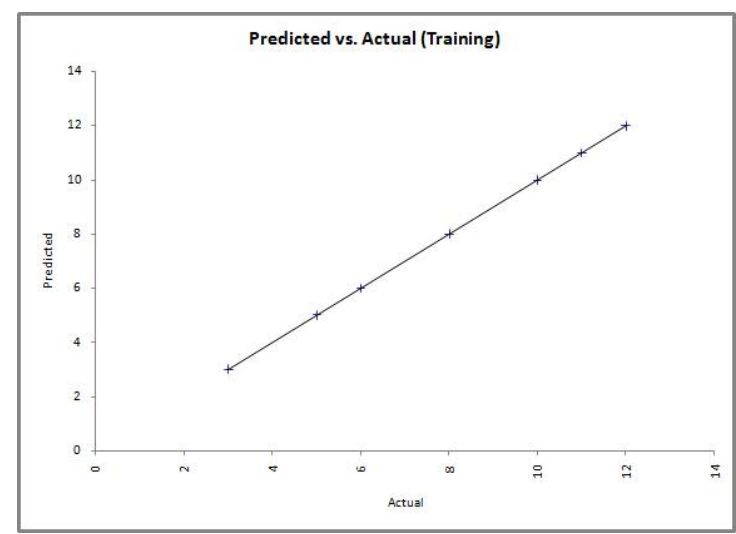

a

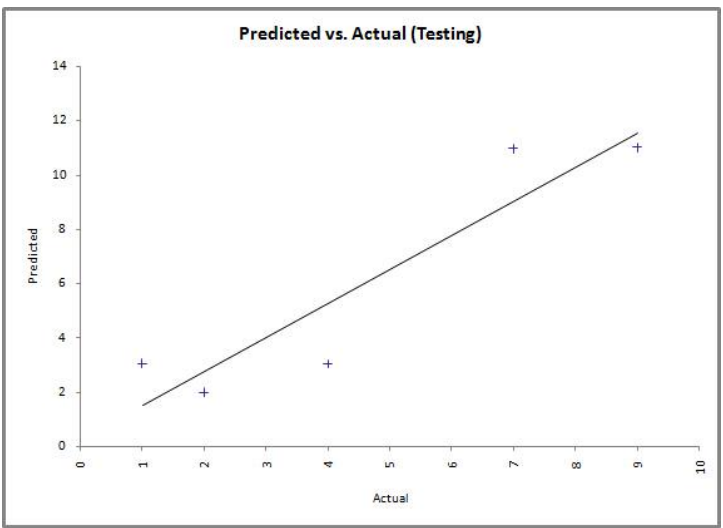

b

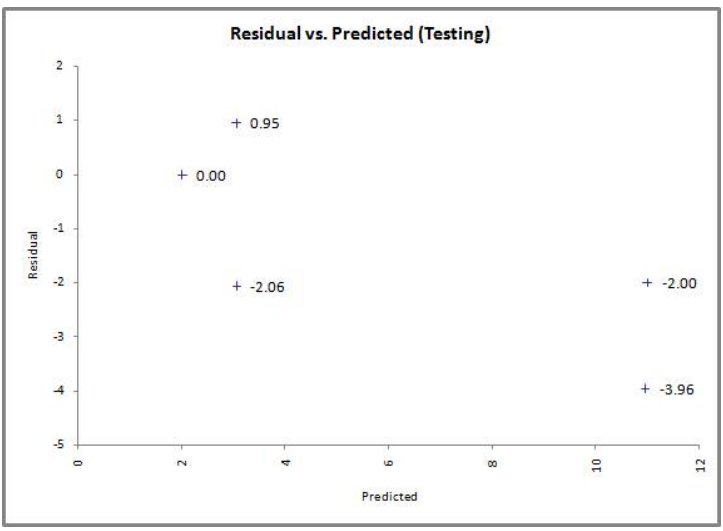

c

Figure 5. Training and Testing Results of MLFN Model with Three Nodes; (a) Predicted Values Versus Actual Values after the Training Process; (b) Predicted Values Versus Actual Values after the Testing Process; (c) Residual Values Versus Predicted Values in Testing Process

Figure 5 shows shat the training and testing results are robust, indicating that the MLFN model with three nodes is very effective for the evaluation in the overall evaluation for vehicles. However, the training time of the ANN models cannot be neglected because there are too many independent variables in this total process of model establishment. Nevertheless, if the total

All results shown by Sections 4.1 to 4.4 shows that ANN models (GRNN and MLFN models with different nodes) are very useful tools that can address the problem of stability 
evaluations for vehicles. With relatively low RMS errors and comparatively high testing results (degree of fitting in training and testing results), we can see that ANN suitable ANN models can generate exact and previous responses during the training process (machine learning process). As for the GRNN model, we can see that it can generate the best results with the lowest RMS errors in many aspects of evaluations for the stability of vehicle handling. As for the MLFN model, we can see that the number of nodes is very important. Different nodes in a MLFN model will greatly influence the results and training time. Using too many nodes in a training process of MLFN model will waste too much time, which is not available in practical applications. Therefore, we strongly encourage that scientists and technicians should make more comparison before making a decision to choose an specific model. As for other models that are not presented in our experiments such as back propagation neural network (BPNN) [20-23] may also available in previous researches. However, the robustness of a BPNN model may not be quite high compared to GRNN model because GRNN model Gaussian function, which has a better fitting ability in related applications. What's more importantly, there are well-packed software that can help scientists and technicians to develop GRNN and MLFN models [24-26], and so we strongly believe that GRNN model and MLFN model would be a better choice in practical applications such as laboratory works and industries.

\section{Conclusion}

Artificial neural networks (ANNs) are of great significance to prediction and evaluation related researches. It can generate correct responses using non-linear fitting principles to mimic human being's thinking patterns. Especially, general regression neural networks (GRNN) and multi-layer feedforward neural network (MLFN) can be widely used because they have been packed into the useful software and they can generate relatively good results for data-prediction. Our work aim at using the principles of ANN models in order to resolve the problems frequently occur in vehicle handling.

In this paper, we present a novel method based on artificial neural networks (ANNs) to aid the evaluation process for the tests of stability of vehicle handling. We set different impact factors of the tests as the independent variables, while the scores of the tests were set as the dependent variables. Using the existing data, we trained it using linear predictor, general regression neural network and multi-layer feedforward network during the machine learning process. Results show that ANN models can be used for aiding the subjective evaluation of stability in vehicle engineering. Results show that GRNN model is the best of the models for snack-like test with an RMS error 0.96, GRNN model and MLFN model with three nodes are both the best of the models for the input test of steering wheel angle pulse, with RMS errors 0.29 and 0.30 respectively, GRNN model is the best of the models for the test of stable circle, with an RMS error 0.45, and MLFN model with three nodes is the best the models for the overall evaluation, with an RMS error 4.23. Our results show that ANN models can be used for the evaluation of stability of vehicle handling. In this article, we aim at providing a novel method for relevant evaluations. The independent variables can be changed according to different circumstances. And we strongly encourage that related scientists can try more different independent variables in the process of model training (machine learning process). In future research, we will concentrate on the application of other machine learning techniques and make comparisons among different advanced modes in the evaluation for stability of vehicle handling.

\section{References}

[1] J. He, D. A. Crolla and M. C. Levesley, SAE Technical Paper, (2004).

[2] X. Yang, Z. Wang and W. Peng, "Vehic. Syst. Dyn.", vol. 47, no. 1, (2009).

[3] M. Shino and M. Nagai, "JSAE review", vol. 22, no. 4, (2001).

[4] R. W. Allen, T. T. Myers and T. J. Rosenthall, "SAE Technical Paper", (2000). 
[5] J. Y. Wu, H. J. Tang and S. Y. Li, “Int. J. Auto. Tech.”, vol. 8, no. 3, (2007).

[6] C. Andrieu, N. De Freitas and A. Doucet, "Machine learning", vol. 50, nos. 1-2, (2003).

[7] F. Sebastiani, "ACM computing surveys (CSUR)", vol. 34, no. 1, (2002).

[8] D. Freitag, "Machine Learning", vol. 39, nos. 2-3, (2000).

[9] J. R. Quinlan, "Machine Learning, vol. 1, no. 1, (1986).

[10] J. Van Schependom, G. Nagels and W. Yu, "Schizophrenia Bulletin “, (2013).

[11] M. Shao, X. J. Zhu and H. F. Cao, "Energy", vol. 67, (2014).

[12] C. H. Aladag, A. Kayabasi and C. Gokceoglu, "Neural Comput. App.", vol. 23, no. 2, (2013).

[13] E. C. Santos, E. D. Armas and D. Crowley, "Soil Bio.”, Bioche., vol. 69, (2014).

[14] H. Yip, H. Fan and Y. Chiang, "Auto. Const.", vol. 38, (2014).

[15] C. M. Hong, F. S. Cheng and C. H. Chen, "Int. J. Elect. Power \& Energy Syst.”, vol. 60, (2014).

[16] D. Svozil, V. Kvasnicka and J. Pospichal, "Chemo. Intel. Lab. Syst.”, vol. 39, no. 1, (1997).

[17] T. D. Sanger, "Neural Networks", vol. 2, no. 6, (1989).

[18] Y. Bai, "Jilin University", (2005).

[19] H. Li, X. F. Liu and S. J. Yang, “Int. J. Electrochem. Sci.”, vol. 9, no. 7, (2014).

[20] P. Wang, X. Ji and L. Zhu, "Sensors and Actuators A: Physical", vol. 201, (2013).

[21] K. Z. Huang, X. K. Xiong and C. M. Zhang, "E Protein and peptide letters", (2014).

[22] P. Wang, L. Zhu and Q. Zhu, "NDT \& E International", vol. 55, (2013).

[23] A. T. C. Goh, “Artificial Intelligence in Engineering”, vol. 9, no. 3, (1995).

[24] L. M. Saadah, F. D. Chedid and M. R. Sohail, "Pharmacotherapy: The Journal of Human Pharmacology and Drug Therapy", vol. 34, no. 3, (2014).

[25] I. Vinnychuk, O. Vinnychuk and L. Skrashchuk, "Intelektiné ekonomika", vol. 8, no. 1, (2014).

[26] L. Lei, L. Song, H. Wang, “J. Chem. Pharm. Res.”, vol. 6, no. 6, (2014).

\section{Authors}

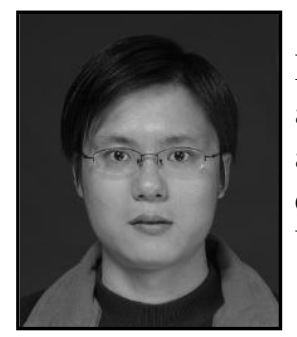

Rui Yu, (1980-08), he was born in Changshu, Jiangsu Province, China, is a lecture in the field of automotive materials and processes, system dynamics automobile, automotive design and dynamic simulation and control. He obtained his master's degree in the Department of vehicle engineering, Nanjing University of Aeronautics and Astronautics.

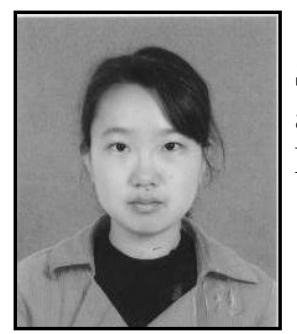

Xiaohui Xia, she is a graduate student in Nanjing University of Science \& Technology, majoring in applied math. She was graduated and obtained the bachelor's degree in College of Applied Math, Nanjing University of Science \& Technology. 